\title{
Aspectos das práticas alimentares e da atividade física como determinantes do crescimento do sobrepeso/obesidade no Brasil
}

\author{
Dietary and physical activity factors \\ as determinants of the increase \\ in overweight/obesity in Brazil
}

\author{
1 Departamento de Nutrição \\ Social, Universidade Federal \\ Fluminense, Niterói, Brasil. \\ 2 Centro de Estudos da Saúde \\ do Trabalhador e Ecologia \\ Humana, Escola Nacional \\ de Saúde Pública, \\ Fundação Oswaldo Cruz, \\ Rio de Janeiro, Brasil. \\ Correspondência \\ Luiz Antonio dos Anjos \\ Rua Leopoldo Bulhões 1480 \\ Rio de Janeiro, $R J$ \\ 21041-210, Brasil. \\ anjos@ensp.fiocruz.br
}

\section{Abstract}

Data from Brazilian national surveys conducted since the 1970s have indicated an increase in the prevalence of overweight/obesity. The two most frequently cited causes of this trend have been dietary changes and reduction in physical activity, characterizing a "contemporary Western lifestyle". The objective of this paper is to identify and assess some indicators associated with changes in eating habits and physical activity patterns in the Brazilian population in the last 30 years. Despite the lack of detailed data, the following factors appear to play an important role in the process: internal migration; dining out; increased consumption of fast food; labor changes; transportation; and the use of home appliances. More information on dietary and physical activity patterns in association with anthropometric data on the Brazilian population are needed for a better understanding of each factor's role in these relationships. Meanwhile, inter-sector programs and projects with well-defined targets and outcomes are needed for the population's adherence to a healthier lifestyle, in order to control the increase in overweight/obesity.

Obesity; Food Habits; Physical Activity
Cristina Pinheiro Mendonça 1

Luiz Antonio dos Anjos 1,2

\section{Introdução}

A obesidade pode ser definida, de forma simplificada, como uma doença caracterizada pelo acúmulo excessivo de gordura corporal, sendo conseqüência de balanço energético positivo e que acarreta repercussões à saúde 1 com perda importante não só na qualidade como na quantidade de vida 2 . Diversos autores $3,4,5$ têm apontado motivos diferentes para o surgimento e a manutenção da obesidade em inúmeras populações. Os estudos que têm sido empreendidos correlacionando aspectos genéticos à ocorrência de obesidade não têm sido capazes de evidenciar a interferência destes em mais de um quarto dos obesos, fazendo com que ainda se acredite que o processo de acúmulo excessivo de gordura corporal, na maioria dos casos, seja desencadeado por aspectos sócio-ambientais 6,7.

Os dois aspectos mais apresentados como relacionados a um quadro de balanço energético positivo têm sido mudanças no consumo alimentar, com aumento do fornecimento de energia pela dieta, e redução da atividade física, configurando o que poderia ser chamado de estilo de vida ocidental contemporâneo 4,8 . Assim, fica claro o papel dos aspectos sócioculturais nesta determinação, formando uma rede de fatores 9 , cuja aproximação vai permitir compreender e intervir no atual quadro em evolução. 
Considerando os aspectos acima, este artigo tem por objetivo identificar e avaliar alguns indicadores que se correlacionam com mudanças nas práticas alimentares e de atividade física, e que permitam compor um quadro com os dados disponíveis sobre sobrepeso/obesidade na população brasileira nos últimos trinta anos. Dentro dessa perspectiva, discute-se a recomendação do Ministério da Saúde do Brasil (MS) para a manutenção do peso saudável.

\section{O quadro de mudanças econômicas e nutricionais}

Especialmente após a Segunda Guerra Mundial, mudanças no perfil epidemiológico com o aumento da prevalência de doenças crônicas não transmissíveis, tais como as doenças cardiovasculares, diversos tipos de câncer, diabetes, e obesidade, nos países do hemisfério norte, propiciaram a ampliação das correlações causais com a alimentação, redução de atividade física e outros aspectos vinculados à vida urbana. Atualmente, estas doenças também são entendidas como problemas de saúde pública nos países do hemisfério sul ou "países pobres", como denominam alguns autores 10,11,12.

Em termos econômicos, o período após 1945, caracterizou-se como "os anos dourados do capitalismo", marcados pela criação de novos mercados e repetidos ganhos de produtividade industrial. Neste período, privilegiava-se o atendimento do mercado de massas com produtos padronizados produzidos em grandes quantidades, e garantindo ganhos em escala e redução de custos, configurando o chamado modelo "fordista" 13. Neste período, sobretudo na Europa Ocidental e América do Norte, verificou-se ampliação na produção industrial de bens de consumo duráveis (especialmente, automóveis e eletrodomésticos, tais como geladeiras e televisores) e pelo aumento acelerado do setor de serviços, além de um período de crescimento do espaço urbano 14.

No final dos anos 60 , os ganhos de produtividade decresceram e a sociedade requisitava produtos cada vez mais segmentados, levando à crise do modelo estabelecido. No âmbito da indústria agroalimentar, as mudanças também foram sentidas, e a partir da década de 80 a diversificação da produção e do consumo permitiu o lançamento de produtos, tais como os semiprontos, étnicos, dietéticos, saudáveis e naturais 13 .
Na economia brasileira, embora o processo de industrialização tenha sido iniciado a partir da década de 30 do século XX, e tenha tido um significativo desenvolvimento a partir dos anos 50 , iniciando inclusive a produção de alguns bens de consumo duráveis, como automóveis, somente a partir de meados da década de 70 é que verificou-se crescimento expressivo da indústria de bens duráveis, em conjunto com o progressivo processo de industrialização da produção do setor primário (agropecuário) e aumento bastante significativo, em diversidade e volume, na produção de bens de consumo não duráveis 15,16.

A industrialização do país fez com que ocorressem modificações importantes no quadro distributivo da mão de obra como redução das ocupações nos setores extrativos e da agricultura em toda a população e que, no caso das mulheres, foi acompanhada de aumento nos setores do comércio e sociais e leve redução do setor de serviços, padrão de mudança que será discutido mais tarde. Esse processo expandiu a migração interna: em 1970 os moradores das áreas urbanas representavam $58,0 \%$ da população, já em 2000, segundo os dados do Censo, $81,0 \%$ da população residia nos aglomerados urbanos 17,18 .

Acompanhando este deslocamento, os dados sobre o sobrepeso/obesidade na população brasileira vêm demonstrando crescimento da sua prevalência entre as décadas de 70 e 90. As análises comparativas entre os inquéritos antropométricos nacionais (Estudo Nacional de Despesa Familiar - ENDEF -, realizado nos anos de 1974/1975 e a Pesquisa Nacional sobre Saúde e Nutrição - PNSN - de 1989) e regionais (Pesquisa de Padrões de Vida - PPV - de 1997), permitiram identificar a amplitude e gravidade do problema. A tendência encontrada para o sobrepeso (IMC $\geq 25 \mathrm{~kg} . \mathrm{m}^{-2}$ ) nos adultos ( $\geq 20$ anos) foi a de aumento na prevalência entre os três estudos, com esta chegando a ser o dobro em 1997 em relação a 1975, com exceção das mulheres no Sudeste (Figura 1).

Ao se incluir estratificação por renda, o quadro apresenta algumas mudanças, tais como: a obesidade (IMC $\geq 30 \mathrm{~kg} \cdot \mathrm{m}^{-2}$ ) ter aumentado em todos os quartis de renda entre as mulheres do Nordeste e ter declinado para os três quartis mais elevados de renda das mulheres do Sudeste. A obesidade entre as mulheres da Região Sudeste só apresentou elevação entre aquelas que compõem os $25,0 \%$ mais pobres, passando de $11,6 \%$ em 1989 , para $15,0 \%$ em 1997. A obe- 
Figura 1

Prevalência de sobrepeso (índice de massa corporal $>25 \mathrm{~kg} \cdot \mathrm{m}^{-2}$ ) em adultos (idade $\geq 20$ anos)

brasileiros das regiões Nordeste e Sudeste em três inquéritos: ENDEF/1975; PNSN/1989; e PPV/1997.

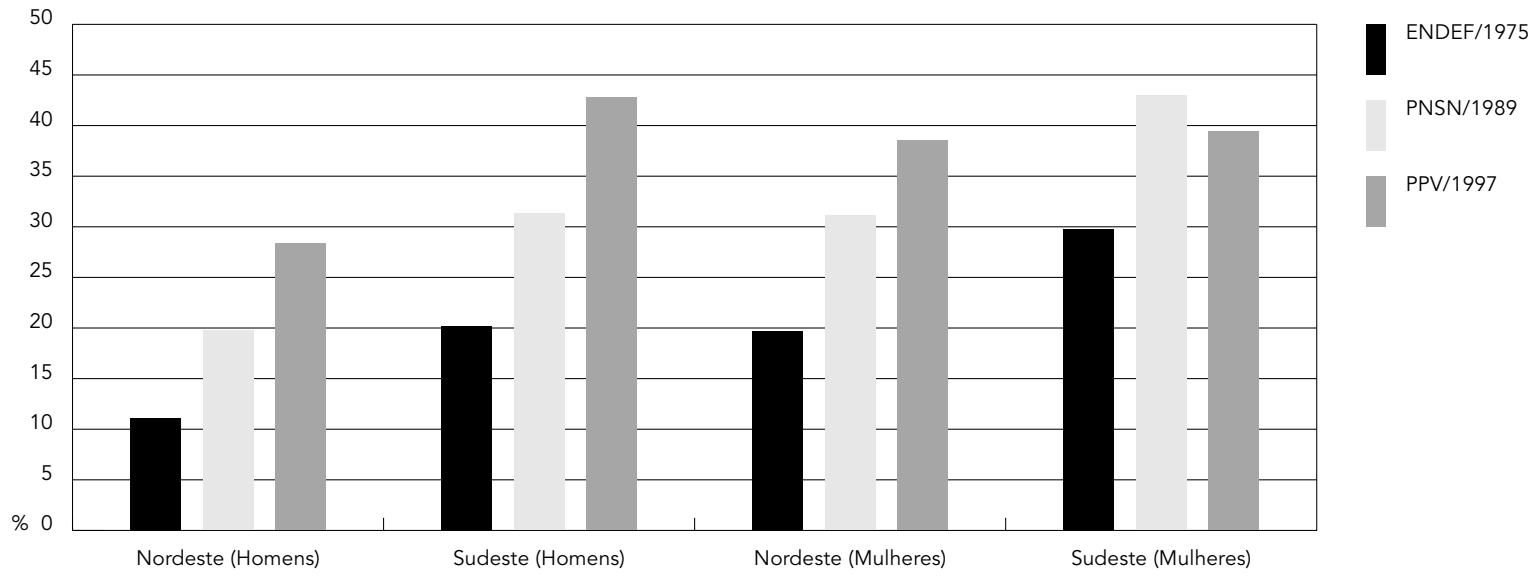

sidade entre os homens, entre 1989 e 1997, cresceu em todos os quartis de renda nas duas regiões 19 .

Os dados destes estudos evidenciam que o problema do sobrepeso/obesidade nos brasileiros adultos afeta, proporcionalmente, quase o dobro de mulheres em relação aos homens, de certa forma repetindo o que ocorria com o baixo peso, pois em 1974/1975 o baixo peso atingia $6,8 \%$ dos homens e $10,4 \%$ das mulheres, e mesmo tendo-se reduzido estes percentuais $(3,4 \%$ e $6,1 \%$, em 1997$)$, a diferença entre os sexos foi mantida 20 .

\section{As relações com as práticas alimentares}

Ao se focalizar a obesidade pelos aspectos vinculados a alterações na dieta, cabe destacar que o aumento da ingestão energética pode ser decorrente tanto da elevação quantitativa do consumo de alimentos como de mudanças na dieta que se caracterizem pela ingestão de alimentos com maior densidade energética, ou pela combinação dos dois. O processo de industrialização dos alimentos tem sido apontado como um dos principais responsáveis pelo crescimento energético da dieta da maioria das populações do Ocidente 21,22,23.

No que diz respeito ao consumo alimentar da população brasileira, não há pesquisas de base populacional, em nível nacional, que permitam acompanhar as mudanças efetivamente ocorridas nas décadas de 1980 e 1990. Análises empreendidas com base nos dados de Pesquisas de Orçamentos Familiares (POF) realizadas pelo IBGE (Fundação Instituto Brasileiro de Geografia e Estatística) nos anos de 1988 e 1996 apontam tendência de crescimento na aquisição de alimentos ricos em lipídeos nas regiões Norte e Nordeste e elevação dos carboidratos simples, acompanhada de redução na aquisição de alimentos fonte de carboidratos complexos. Este quadro se configura por conta do aumento na aquisição de carnes, de leite e de seus derivados, de açúcar e refrigerantes e do declínio nas compras de leguminosas, hortaliças e frutas 24 .

Embora a concentração de renda no Brasil (as classes de renda mais baixa utilizam $37,0 \%$ da renda com alimentação e as mais altas empenham só $11,0 \%$ ) não permita falar em mercado homogêneo, nas duas últimas décadas houve um predomínio no consumo de alimentos industrializados comprados em supermercados em todas as classes de renda 13,25. As mudanças verificadas por intermédio das POF indicam incremento na aquisição de produtos industrializados e redução de alimentos in natura por parte das famílias, pois as carnes, especialmente o frango, e os laticínios têm tido uma enorme ampliação na oferta de produtos processados e os refrigerantes constituem em si, a representação máxima da industrialização na área de alimentos e bebidas. Castro \& Peliano 26 apontam que preço, paladar e nutrição 
aparecem como critérios de decisão para a inclusão de alimentos nas práticas alimentares, mas sempre intermediados por um filtro cultural. Hoje, nos supermercados da maioria das cidades brasileiras é possível adquirir alimentos resfriados, congelados, temperados, preparados, empanados, recheados, em forma de hambúrguer, almôndegas etc., e a maior parte dos produtos tem como indicação de cozimento, a fritura. Dessa forma, o aumento da concentração energética pode se dar pelos recheios, molhos, temperos acrescentados aos produtos e pelo modo de preparo. Cyrillo et al. 25 destacam o crescimento acentuado na comercialização de mistura para bolo, iogurte, queijos petit suisse, sobremesa pronta gelificada, suco de fruta concentrado ou pronto para consumo no período entre 1994 e 1996, contribuindo para reforçar as possibilidades já apresentadas para o aumento da densidade energética da dieta.

Um dado curioso exposto por Monteiro et al. 24 é a redução da energia diária per capita disponível entre os dois levantamentos das POF do IBGE (1988 e 1996), quando passou-se de $1.919,0 \mathrm{kcal}$ para $1.711,2 \mathrm{kcal}$ nas regiões metropolitanas brasileiras. Aparentemente, o consumo estaria na contramão do aumento na prevalência da obesidade; porém, convém lembrar que algumas questões importantes na estimação do consumo alimentar não foram levantadas nas duas POF. Primeiramente os fluxos não monetários não foram avaliados e, portanto, não incluídos nos indicadores de compra, fato que acarreta subestimativa do consumo alimentar dependendo do grau de monetarização da região em estudo. Além disso, a estimativa do consumo energético feito em refeições fora de casa pode comprometer o cálculo final do consumo energético, dependendo da importância relativa desse consumo em relação ao consumo energético total. Em estudo comparativo realizado nos Estados Unidos entre 1977 e 1985, observou-se que a energia da dieta vinda da alimentação feita fora do domicílio aumentou de $18,0 \%$ do total consumido em 1977 para 34,0\% em 1995, e como vem se confirmando a observação de que a alimentação preparada fora do domicílio contém maior densidade energética, a avaliação do consumo realizado fora de casa ganha destaque ainda maior quando a preocupação está no avanço acelerado na prevalência da obesidade 23 .

No setor industrial agroalimentar brasileiro, mudanças começaram a ocorrer nos anos 70 e se consolidaram nos anos 80 , potencializando um mercado urbano e jovem, o que pode ser exemplificado pelo crescimento das despesas com alimentação fora de casa, particu- larmente em restaurantes do tipo fast food e com a alimentação em locais de trabalho ou em bares e restaurantes com a utilização de vale-refeição 20 . Uma rede internacional de alimentação rápida se vangloria de ter o Brasil, hoje, entre os oito maiores mercados internacionais da corporação, com 570 restaurantes, cerca de 640 quiosques e 17 McCafés (http:// www.mcdonalds.com.br). O faturamento da empresa no país, em 2001, foi de R $\$ 1,60$ bilhão com o atendimento de 514 milhões de clientes - média de 1,4 milhão de clientes a cada dia. $\mathrm{O}$ Brasil está entre os cinco países que mais cresceram nos últimos anos entre todos os mercados da corporação no planeta. De fato, o crescimento em termos de número de lojas é exponencial desde sua introdução no país em 1979, sobremodo a partir dos anos 90 (Figura 2). A introdução desses hábitos importados além de comprometer o padrão tradicional alimentar no país faz com que ele seja alterado com substituição de refeições. Essas modificações podem contribuir para a dificuldade na manutenção da massa corporal dentro de padrões considerados saudáveis. Por exemplo, Sichieri 27 demonstrou, em inquérito de base domiciliar na Cidade do Rio de Janeiro, que um padrão dietético, por ela chamado de tradicional e que dependia principalmente de feijão com arroz, estava associado com risco menor, em adultos, de sobrepeso/obesidade em modelo logístico ajustado para idade e atividade física de lazer em comparação a uma dieta chamada de ocidental na qual a gordura e açúcar eram os principais componentes, ou uma dieta de padrão misto na qual não havia um alimento que se destacasse.

Associado ao fato de não existirem medidas antropométricas nas duas POF, o que não permite a estimativa de requerimento energético, a informação sobre o consumo energético declinante entre as duas pesquisas é questionável. Em verdade, a análise do banco de dados da folha de balanço alimentar do Brasil disponibilizado pela Organização das Nações Unidas para a Alimentação e Agricultura (FAO) indica que a quantidade de energia per capita disponível para consumo humano aumentou substancialmente nas últimas décadas no país, passando de aproximadamente 2.200 para $3.000 \mathrm{kcal} /$ habitante/dia entre 1961 e 1999, ou seja, em torno de $188 \mathrm{kcal} /$ dia de acréscimo por década 28. A grande modificação se deu no aumento da contribuição percentual dos lipídeos no total de energia com o aporte de proteína permanecendo estável no período.

Em suma, são vários os fatores associados à dieta que poderiam contribuir para o aumento 
Figura 2

Tendência do número de restaurantes McDonald's desde sua instalação no país.

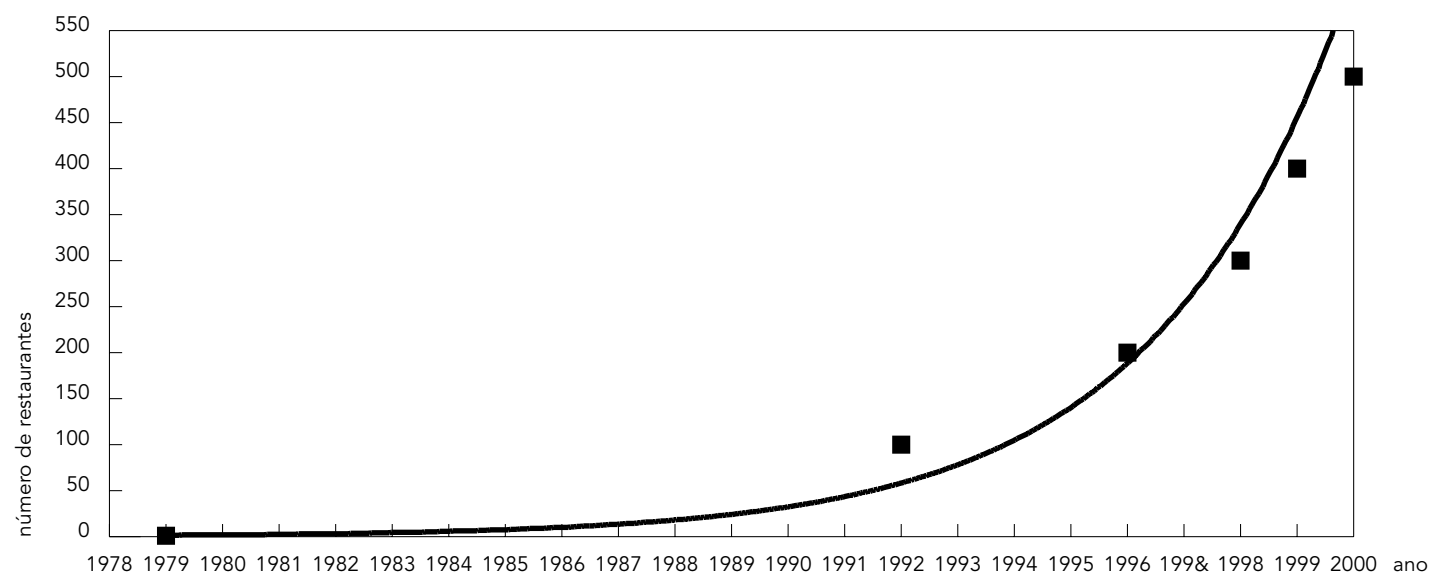

do sobrepeso/obesidade dos brasileiros ao acarretarem mudanças importantes nos padrões alimentares tradicionais: (1) migração interna; (2) alimentação fora de casa; (3) crescimento na oferta de refeições rápidas (fast food); (4) ampliação do uso de alimentos industrializados/processados. Estes aspectos vinculam-se diretamente à renda das famílias e às possibilidades de gasto com alimentação, em particular, associado ao valor sócio-cultural que os alimentos vão apresentando para cada grupo social.

\section{As relações com a atividade física}

O gasto energético, a outra parcela da equação de balanço energético, é resultado de 29: taxa metabólica basal (TMB), que é o gasto para manter em funcionamento as atividades vitais do organismo; ação dinâmica específica dos alimentos, que corresponde ao gasto energético necessário para a digestão, absorção e utilização dos alimentos; e atividade física, que pode ser entendida como qualquer movimento corporal produzido pela contração da musculatura esquelética que implique em gasto energético 30 . A atividade física, então, compreende uma gama de dimensões que incluem todas as atividades voluntárias, como as ocupacionais, de lazer, domésticas e de deslocamento.

A redução do nível de atividade física e sua relação com a ascensão na prevalência da obesidade refere-se às mudanças na distribuição das ocupações por setores (exemplo: da agri- cultura para a indústria) e nos processos de trabalho com redução do esforço físico ocupacional; das alterações nas atividades de lazer, que passam de atividades de gasto acentuado, como práticas esportivas, para longas horas diante da televisão ou do computador; e do uso crescente de equipamentos domésticos com redução do gasto energético da atividade, como por exemplo, lavar roupa à máquina ao invés de fazê-lo manualmente 31 .

Os dados sobre as características de atividade física da população são ainda mais escassos do que sobre o consumo alimentar, uma vez que não existe estudo nacional de base populacional que tenha investigado o nível de atividade física. Até recentemente havia uma tendência, nos países desenvolvidos, de se pesquisar somente a atividade física de lazer pelo fato de o indivíduo ter maior controle dessas atividades por serem atividades voluntárias, facilitando, dessa forma, a obtenção de informações mais confiáveis e também por assumir-se que as atividades ocupacionais nesses países são, na sua grande maioria, leves. Entretanto, o não uso das atividades ocupacionais (e domésticas) em países desenvolvidos é em razão da indisponibilidade de tais informações; mas, hoje em dia, se reconhece que a medição somente da atividade física de lazer leva a uma subestimação da atividade física total, especialmente nos indivíduos com ocupações intensas 32 . Em levantamento realizado em 1990, aproximadamente $50,0 \%$ dos trabalhadores americanos, que não praticavam atividade física de lazer, 
disseram realizar pelo menos uma hora de atividade ocupacional pesada 33 .

No Brasil, numa comparação do nível de atividade física ocupacional (NAFO), baseado nas informações sobre a ocupação principal, entre os dados do ENDEF e da PNSN, percebeu-se que a prevalência do NAFO leve e moderado aumentou, enquanto a do pesado declinou, passando de $25,2 \%$ para $22,6 \%$. Os percentuais de redução das atividades ocupacionais de nível pesado e o ligeiro aumento dos níveis leve e moderado não seriam capazes, isoladamente, de justificar o crescimento tão acelerado e extenso da obesidade entre os adultos brasileiros 31,34 . Na verdade, aconteceu aumento de obesidade em todos os níveis de atividade física. Deve-se ter em mente que essas comparações sofrem limitações porque podem ter havido mudanças tecnológicas nos processos de trabalho entre os estudos e podem existir diferenças substanciais no gasto energético para realizar a mesma tarefa ocupacional entre as várias regiões do país.

Entre os censos de 1960 e 1991, houve, entre os homens com mais de dez anos de idade, tendência de aumento das atividades dos setores da indústria e serviços e redução na agricultura e extração (Figura 3). Os setores que mais têm empregado mulheres são os de prestação de serviços, seguidos pelo agrícola e comercial, sendo a prestação de serviços e o comércio correspondentes, em 1991, a aproximadamente $47,0 \%$ dos setores de ocupação das mulheres, seguido do setor social com $20,0 \% 17,35,36,37$. A grande modificação na distribuição dos setores de ocupação até o censo de 1991 foi a redução do trabalho agrícola e o aumento das atividades sociais e do comércio. É interessante notar que o crescimento da participação feminina na população ativa ocupada de maiores de dez anos ocorreu de modo concomitante ao aumento da obesidade entre as mulheres. Em 1960 , as mulheres ocupadas representavam $16,5 \%$ da população ativa com mais de dez anos, percentual que aumentou para $31,0 \%$ no Censo de 1991, chegando a 43,6\% em 2000. Por outro lado, houve redução nesse percentual para os homens, de $77,2 \%$ em 1960 para aproximadamente $68,0 \%$ entre os dois últimos censos $17,18,35,36,37$.

Os indicadores fazem crer que o aumento da participação feminina no mercado de trabalho foi mais acentuado, nas últimas décadas, para aquelas de estratos sócio-econômicos médio e alto, e em atividades que provavelmente poderiam ser consideradas como leves do ponto de vista de gasto energético, pois se verifica um incremento nos anos de estudo e no rendi- mento médio do trabalho feminino ${ }^{18}$. Infelizmente, os dados não permitem análises de tendências tanto nas atividades informais quanto nas domésticas, já que não há séries históricas dessas informações nos censos. Pesquisas locais evidenciam essa característica. Rotenberg 38 documentou, em mulheres moradoras da Favela da Rocinha, no Rio de Janeiro, uma série de atividades remuneradas realizadas no espaço doméstico, tais como lavar roupa para fora e tomar conta de crianças da vizinhança, que não eram consideradas pelas próprias mulheres entrevistas como trabalho, e que provavelmente não as registravam por ocasião dos recenseamentos.

Com relação às atividades não ocupacionais (lazer, transporte, doméstica) não se realizou, até o momento, pesquisa de base domiciliar e de abrangência nacional que as tenha investigado com detalhe 34 . Na PPV, $20,0 \%$ da população indicou a prática de exercício físico ou esporte (EF), havendo um grande diferencial entre homens $(27,3 \%)$ e mulheres $(13,1 \%)$. A freqüência de prática de EF foi semelhante entre as regiões Nordeste (18,7\%) e Sudeste $(20,9 \%)$; contudo, substancialmente menos mulheres do Nordeste $(8,9 \%)$ praticavam EF comparadas às do Sudeste $(15,9 \%)$. Entre as pessoas que não faziam EF, 23,0\% e 49,3\% tinham NAFO pesado e moderado, respectivamente. Ao somarem-se as pessoas com NAFO pesado às pessoas que disseram que não praticavam EF, o total de ativos sobe para $27,7 \%$ e para $44,2 \%$, se forem acrescentadas as com NAFO moderado 39. Apesar da limitação na pergunta sobre a prática de EF, esta foi a primeira pesquisa de base populacional em regiões brasileiras que obteve tal informação.

Retomando aspectos vinculados ao desenvolvimento da economia no Brasil, a aquisição de bens de consumo duráveis por parte da população pode contribuir para a consideração de três fatores ligados a mudanças no padrão de atividade física enquanto redução do gasto energético: (1) diminuição do esforço com o trabalho doméstico pelo uso de equipamentos para a execução das tarefas mais árduas; (2) o crescente uso da televisão como principal meio de lazer; e (3) o uso de automóvel/veículo automotivo para o deslocamento. Deve-se ainda acrescentar que a televisão contribui para a delimitação do estilo de vida ocidental mediante ampliação do incentivo ao consumo difundido pelo marketing. De acordo com French et al. 23, nos Estados Unidos, em 1997, os restaurantes fast foods gastaram $95,0 \%$ de suas verbas publicitárias com propagandas na televisão, e o volume de dólares gastos pela indústria de ali- 
Figura 3

Distribuição (\%) das pessoas com 10 anos ou mais de idade, ocupadas por setores

de atividade do trabalho principal nos Censos Demográficos Brasileiros.

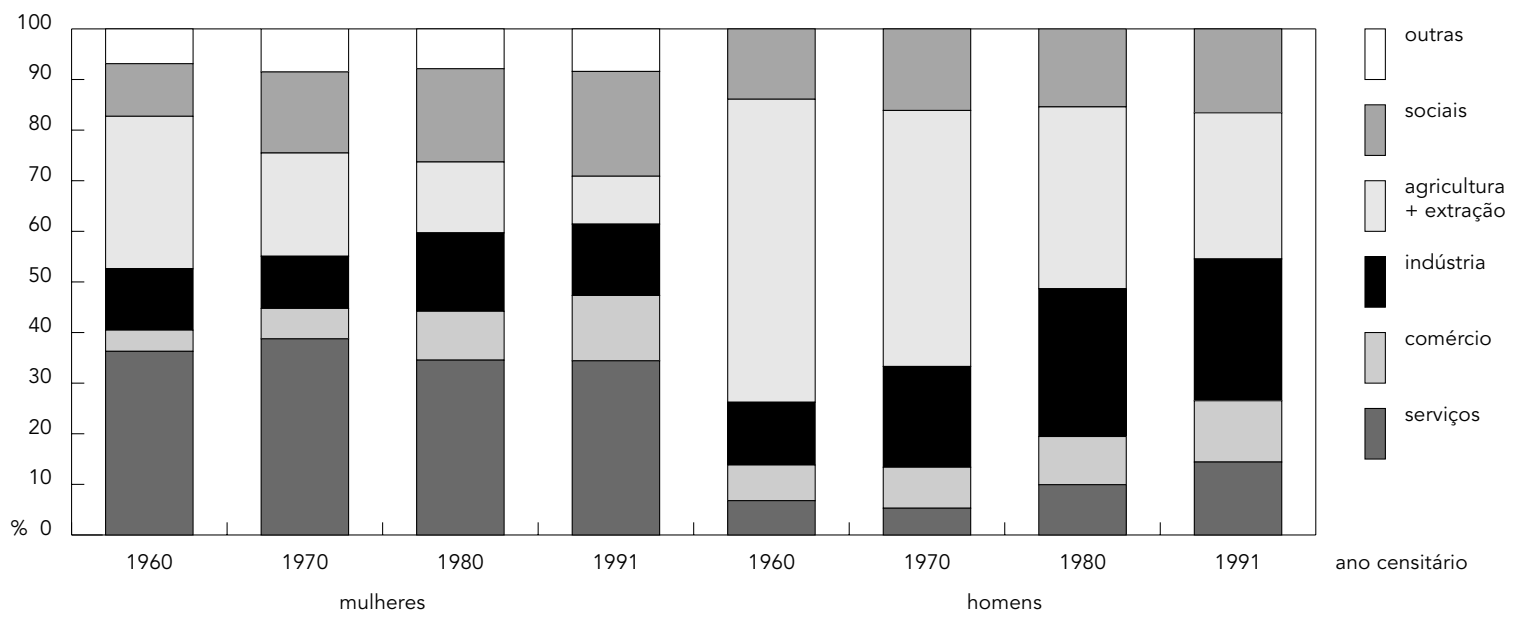

mentos e por serviços de alimentação com publicidade, só ficou atrás daqueles utilizados pela indústria automobilística, a primeira do ranking. Segundo Belik 13, a embalagem e o marketing estão entre os principais componentes na estrutura de custos dos produtos atualmente no Brasil.

A observação da tendência do percentual de domicílios com alguns bens duráveis selecionados obtida nos censos demográficos nacionais realizados nas últimas três décadas $17,18,37,38$ indica aumento vertiginoso de residências com televisões e geladeiras e um incremento bem menos acentuado de domicílios com automóveis (Figura 4). O percentual de domicílios com geladeira passou de $26,0 \%$ em 1970 para $83,0 \%$ em 2000. Já o percentual de domicílios com automóveis embora tenha mais do que triplicado, passando de $9,0 \%$ em 1970 para $32,0 \%$ em 2000 , ainda não é tão significativo para o conjunto da população como se tornou a televisão que estava presente em $24,1 \%$ dos domicílios em 1970 , tendo crescido para $56,1 \%$ em 1980 e saltado para $87,0 \%$ no último censo de 2000 .

Com relação ao hábito de assistir à televisão, existe uma tendência atual de utilizar o tempo em horas diárias diante de uma televisão como indicador de vida sedentária, explicando, dessa maneira, a epidemia da obesidade. De fato, essa variável é de fácil obtenção e tem lógica, do ponto de vista explicativo, ao incorporar um hábito que poderá diminuir a prá- tica esportiva de lazer e aumentar o consumo energético, particularmente em crianças/adolescentes. Em inquérito domiciliar americano de base populacional (NHANES III), a prevalência de obesidade foi a menor; entretanto, só significativa para as meninas, entre as crianças que assistiam até uma hora por dia de televisão e a maior naquelas que assistiam até quatro ou mais horas por dia 40 . Não houve associação entre a prevalência de obesidade e a participação semanal em atividade física de nível intenso, tanto em meninos como em meninas. Ou seja, a associação entre a prevalência de obesidade e o tempo sentado assistindo à televisão não pode ser explicada somente pelo aumento da ingestão energética ou redução da prática de atividade física, talvez, em parte, pelos métodos usados na avaliação.

Os deslocamentos diários das pessoas podem contribuir para um estilo de vida ativo ou não. Estimativas de 1995 para a população americana dão conta de que aproximadamente $90,0 \%$ dos deslocamentos eram feitos por automóvel que, em $68,0 \%$ dos casos, incluíam um único ocupante 41 . A opção pelo transporte individual é um dos pilares do sonho americano que é evidenciado pelo número extremamente baixo ( $\cong 5,0 \%$ ) de domicílios que não tinham automóveis nos dados recentes do censo americano ${ }^{42}$. No Brasil, como em muitos outros países, os meios de transporte mais utilizados ainda são os públicos/coletivos ou os não motori- 
Tendência na distribuição (\%) de domicílios brasileiros com televisão, geladeira

e automóvel nos Censos Demográficos Brasileiros.

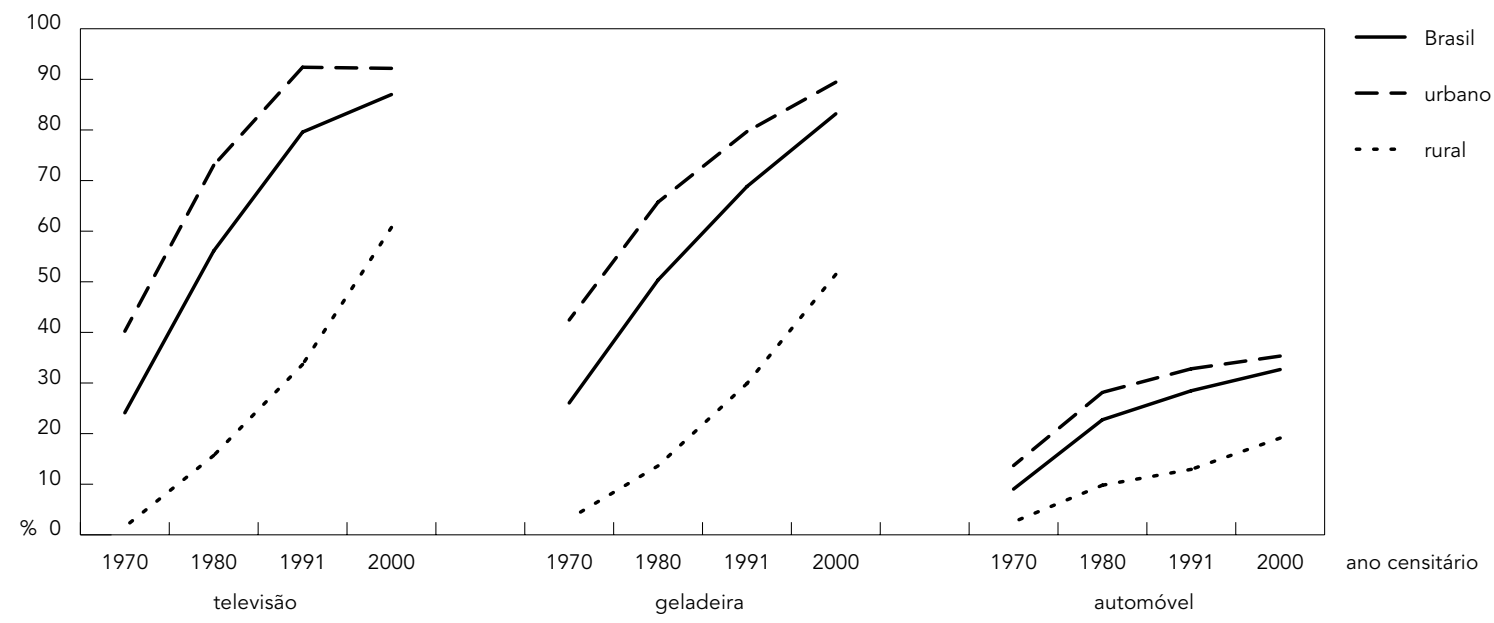

zados, fazendo com que, teoricamente, haja dispêndio maior de energia, mas infelizmente não existem estimativas desse componente do gasto energético para a população brasileira.

Assim como sobre as práticas alimentares, há necessidade de informações mais detalhadas sobre o padrão de atividade física da população brasileira, em associação a dados antropométricos, para uma melhor explicação dessa relação. Apesar da carência de tais informações, o MS vem propondo medidas preventivas para a manutenção do peso saudável na população brasileira. Uma das medidas é a divulgação dos dez passos para o peso saudável, detalhados a seguir.

\section{Os 10 passos para o peso saudável do Ministério da Saúde do Brasil}

Com base na prevalência crescente do sobrepeso/obesidade no país, o MS produziu os dez passos para o peso saudável dentro do Plano Nacional para a Promoção da Alimentação Adequada e do Peso Saudável 43 cujos objetivos são: (1) aumentar o nível de conhecimento da população sobre a importância da promoção à saúde e de se manter peso saudável e de se levar uma vida ativa; (2) modificar atitudes e práticas sobre alimentação e atividade física; (3) prevenir o excesso de peso. Os passos são:

1. Comer frutas e verduras variadas, pelo menos duas vezes por dia;
2. Consumir feijão pelo menos quatro vezes por semana;

3. Evitar alimentos gordurosos como carnes gordas, salgadinhos e frituras;

4. Retirar a gordura aparente das carnes e a pele do frango;

5. Nunca pular refeições: fazer três refeições e um lanche por dia. No lanche escolher uma fruta;

6. Evitar refrigerantes e salgadinhos de pacote; 7. Fazer as refeições com calma e nunca na frente da televisão;

8. Aumentar a sua atividade física diária. Ser ativo é se movimentar. Evitar ficar parado, você pode fazer isto em qualquer lugar;

9. Subir escadas ao invés de usar o elevador, caminhar sempre que possível e não passar longos períodos sentado assistindo à TV;

10. Acumular trinta minutos de atividade física todos os dias.

Os sete primeiros passos estão relacionados à dieta e os três últimos ao incentivo a se ter uma vida mais ativa. Os passos relativos à dieta seguem uma orientação geral de várias instituições estrangeiras, com adaptações locais, como por exemplo, no caso brasileiro, a recomendação da ingestão de feijão pelo menos quatro vezes por semana. Do ponto de vista operacional, as recomendações são baseadas na idéia do guia alimentar americano, popularizada como a pirâmide alimentar, produzida pelo Departamento de Agricultura dos Estados Unidos na expectativa de que quem a se- 
guisse se tornaria mais saudável, ou seja, a mudança no hábito alimentar preveniria o aparecimento de doenças crônicas, pressuposto que vem sendo questionado por dados recentes 44 .

A lógica da pirâmide americana, e que foi adaptada para a população brasileira 45 e é usada como orientação aos consumidores na rotulagem dos alimentos 46 , é baseada na idéia de que os alimentos que estão no topo da pirâmide (óleos, gorduras, açúcares e doces) devam ser consumidos num número menor de porções (1 a 2 por dia) e os da base da pirâmide (grupo dos cereais, pães, farinhas, massas, tubérculos e raízes) devam ser consumidos em número maior de porções (5 a 9 por dia). Existem alguns problemas com essa lógica: (1) da forma que está colocada, passa-se a impressão de que toda gordura é maléfica 47 e transfere-se para o consumidor a modulagem de seu comportamento alimentar 44; (2) a introdução do conceito de porção que não é facilmente assimilado pela população. A questão do tamanho de porção pode ser considerada como um ponto crucial mesmo nos Estados Unidos, já que está ocorrendo uma tendência de aumento substancial no número de porções maiores (large size) no mercado a partir de 1980 48, particularmente nos alimentos industrializados prontos para consumo. O tamanho da porção de um produto na prateleira dos mercados pode ser até sete vezes maior do que a porção definida pelos órgãos responsáveis por sua padronização nos Estados Unidos: Administração de Alimentos e Drogas (FDA) e o Departamento de Agricultura (USDA) 48. Essa tendência tem fundamentos, basicamente, econômicos cujas causas são o aumento na freqüência de refeições realizadas fora de casa, pela competição econômica entre produtores de alimentos (levar mais por um custo menor) e no aumento na oferta de produtos pelo mercado (produtos maiores chamam mais atenção) Para o consumidor, tamanho de porção maior poderá significar ingestão energética de até $30,0 \%$ a mais numa refeição, comparativamente a uma porção menor 49. Esse fenômeno não pode ser ignorado pelos formuladores de políticas públicas de prevenção e tratamento da obesidade.

Cabe ainda comentar a orientação, geral, de nunca pular uma refeição já que esta conflita com a evidência de que o aumento do sobrepeso/obesidade, atualmente, é mais intenso entre os mais pobres, segmento da população em que o acesso a alimentos não é contínuo e nem é caracterizado como padrão regular de refeições.

Os três últimos passos recomendados dizem respeito à prática de atividade física regular com a reprodução da recomendação hege- mônica atual de se acumular pelo menos trinta minutos de atividade física moderada, preferencialmente todos os dias da semana. Essa recomendação foi introduzida inicialmente em 1995 para a população americana adulta 50 na perspectiva de que tal prática acarretasse dispêndio de $1.000 \mathrm{kcal}$ por semana $(200 \mathrm{kcal}$ por dia), valor compreendido como necessário para reduzir substancialmente a taxa de mortalidade comparativamente a uma população que não praticava nenhuma atividade física de lazer 51,52, apesar da maior redução na taxa de mortalidade ter sido encontrada nos indivíduos com gasto energético equivalente a 2.000kcal por semana 53 . O nível de $1.000 \mathrm{kcal}$ por semana é atualmente reconhecido como muito pequeno para a manutenção de massa corporal saudável 54 e possivelmente para a redução do risco relativo de mortalidade por doenças cardiovasculares 55. Considerando a tendência crescente na prevalência da obesidade na população americana e pela sua responsabilidade na produção de recomendações de ingestão dietética de referência (DRI), o Instituto de Medicina Americano do Comitê de Alimentação e Nutrição recentemente sugeriu 56 que a população americana realizasse pelo menos sessenta minutos de atividade moderada (caminhar/ corrida entre 6 e $9 \mathrm{~km} / \mathrm{h}$ ), para a manutenção da massa corporal saudável e para se obter os benefícios para a saúde da atividade física independentes da massa corporal. Essa recomendação se aproxima do valor de NAF (nível de atividade física = gasto energético de $24 \mathrm{~h} /$ taxa metabólica basal de $24 \mathrm{~h} 57$ ) de 1,6 , valor consistente com estilo de vida ativo baseando-se em estudos de água duplamente marcada 56.

Duas importantes recomendações recentes 58,59 concluem que para a manutenção de valores de massa corporal saudável seria necessário um estilo de vida ativo (ou moderadamente ativo), o que implicaria um NAF de 1,75. Para um indivíduo sedentário alcançar esse nível e ajudar na manutenção da massa corporal e prevenção de alguns tipos de câncer, os órgãos mencionados sugerem sessenta minutos de exercício físico diário. Assim, as recomendações recentes convergem para um nível de atividade física maior do que o acúmulo de trinta minutos diários de atividade física moderada.

\section{Considerações finais}

Após apresentação do quadro evolutivo da obesidade no Brasil, percebe-se que, quanto aos fatores determinantes deste processo, o quadro está no esboço que só será elucidado quan- 
do informações detalhadas tanto de ingestão quanto de gasto energético da população sejam obtidas. Esses dados poderão esclarecer o fenômeno inédito e surpreendente da redução na prevalência de sobrepeso/obesidade nas mulheres do sudeste brasileiro.

Quanto às práticas alimentares, é necessário buscar uma maior compreensão do peso da alimentação fora do domicílio e do consumo de produtos industrializados e pré-processados pelos vários segmentos da sociedade. A real dimensão da presença feminina no trabalho fora do lar para as classes média e alta e a concomitante contratação do emprego doméstico por parte destas mesmas mulheres pode contribuir para o delineamento das reais modificações no preparo de alimentos no âmbito domiciliar, no perfil de compra de alimentos por parte das famílias e no consumo de refeições preparadas e/ou servidas fora do domicílio por toda a família, e não apenas por parte das mulheres que se engajam nos postos de trabalho.

As relações entre trabalho feminino fora do domicílio, atividades de lazer, e atividades domésticas também precisam ficar mais claras para que se possa confirmar a possibilidade de diminuição do gasto energético proporcional ao crescimento da prevalência da obesidade entre os adultos brasileiros, uma vez que, à primeira vista, pode parecer que o trabalho doméstico contribui mais para o gasto energético do que o trabalho fora de casa, fato que sem dúvida precisa ser melhor investigado.

Por fim, paralelamente à continuidade na busca de dar maior nitidez a este quadro, há necessidade de que sejam propostos e implementados projetos e programas intersetoriais por parte dos governos e das organizações sociais e que tenham metas bem definidas, que levem a uma redução na velocidade do crescimento da prevalência do sobrepeso/obesidade, por intermédio da conjugação de ações individuais e coletivas na efetivação de uma vida mais saudável para toda a população. A simples divulgação e difusão de uma lista de passos a serem seguidos pelos indivíduos não garante a adesão da população a um estilo de vida saudável, e pior ainda, não consegue atingir os diversos segmentos da sociedade que têm características, motivações e possíveis causalidades diferentes para a obesidade.

\section{Resumo}

Os dados acerca do sobrepeso/obesidade na população brasileira demonstram um crescimento na sua prevalência entre as décadas de 70 e 90. Os dois aspectos mais apresentados como relacionados a este quadro têm sido mudanças no consumo alimentar, com aumento do fornecimento de energia pela dieta e redução da atividade física, configurando um "estilo de vida ocidental contemporâneo". Este artigo tem por objetivo identificar e avaliar alguns indicadores que se correlacionam com mudanças nas práticas alimentares e de atividade física na população brasileira nos últimos trinta anos. Apesar da carência de dados detalhados, os fatores que podem estar contribuindo para o quadro em tela incluem: migração interna; alimentação fora de casa; crescimento na oferta de refeições rápidas; mudanças no trabalho; meios de deslocamento; e equipamentos domésticos. Faltam informações mais detalhadas sobre as práticas alimentares e o padrão de atividade física no Brasil, em associação a dados antropométricos, para uma melhor explicação dessa relação. Há necessidade de projetos e programas intersetoriais que tenham metas bem definidas e que garantam a adesão da população a um estilo de vida saudável na perspectiva do controle do crescimento da prevalência do sobrepeso/obesidade no país.

Obesidade; Hábitos Alimentares; Atividade Física

\section{Colaboradores}

L. A. Anjos foi responsável pelas análises da tendência na situação nutricional antropométrica da população brasileira e pelo levantamento e compilação dos dados censitários. Os autores são responsáveis por todos os aspectos de revisão, preparação e escrita. Coube à C. P. Mendonça o detalhamento dos aspectos nutricionais e a L. A. Anjos os aspectos referentes à prática da atividade física.

\section{Agradecimentos}

L. A. Anjos recebe bolsa de produtividade do Conselho Nacional de Desenvolvimento Científico e Tecnológico (CNPq - proc. 301076/89-8). 


\section{Referências}

1. World Health Organization. Obesity: preventing and managing the global epidemic. Geneva: World Health Organization; 2000. (WHO Technical Report Series, 894)

2. Fontaine KR, Redden DT, Wang C, Westfall AO, Asslison DB. Years of life lost due to obesity. JAMA 2003; 289:187-93.

3. Gigante D, Barros F, Post C, Olinto M. Prevalência de obesidade em adultos e seus fatores de risco. Rev Saúde Pública 1997; 31:236-46.

4. Kumanyika SK. Minisymposium on obesity: overview and some strategic considerations. Annu Rev Public Health 2001; 22:293-308.

5. Sichieri R. Epidemiologia da obesidade. Rio de Janeiro: Eduerj; 1998.

6. Bouchard C. Current understanding of the etiology of obesity: genetic and nongenetic factors. Am J Clin Nutr 1991; 53 (6 Suppl):1561S-5S

7. Stunkard AJ. Factores determinantes de la obesidad: opinión actual, In: La obesidade en la pobreza: un nuevo reto para la salud pública. Washington DC: Organización Panamericana de la Salud; 2000. p. 27-32. (Publicación Científica 576)

8. Garcia RWD. Representações sociais da alimentação e saúde e suas repercussões no comportamento alimentar. Physis (Rio J) 1997; 7:51-68.

9. Sobal J. Obesity and socioeconomic status: a framework for examining relationships between physical and social variables. Med Anthropol 1991; 13:231-47.

10. Mondini L, Monteiro CA. Mudanças no padrão de alimentação. In: Monteiro CA, organizador. Velhos e novos males da saúde do país. São Paulo: Editora Hucitec/Núcleo de Pesquisas Epidemiológicas em Nutrição e Saúde, Universidade de São Paulo; 2000. p. 79-89.

11. Visscher TLS, Seidell JC. The public health impact of obesity. Annu Rev Public Health 2001; 22:35575.

12. Popkin BM. The nutrition transition and obesity in the developing world. J Nutr 2001; 131:871S-3S

13. Belik W. A reestruturação da indústria agroalimentar e abastecimento: uma nova agenda para discussão. In: Galeazzi MAM, organizador. Segurança alimentar e cidadania. Campinas: Mercado das Letras; 1996. p. 295-300.

14. Brum A. O desenvolvimento econômico brasileiro. Petrópolis: Editora Vozes/Ijuí: Fundação de Integração, Desenvolvimento e Educação do Noroeste do Estado; 1991.

15. Oliveira F. O terciário e a divisão social do trabalho. Estudos Cebrap 1979; 24:137-68.

16. Singer P. A economia dos serviços. Estudos Cebrap 1979; 24:127-35.

17. Fundação Instituto Brasileiro de Geografia e Es tatística. Censo Demográfico de 1970. VIII recenseamento geral do Brasil, série nacional, volume I. Rio de Janeiro: Fundação Instituto Brasileiro de Geografia e Estatística; 1973.

18. Fundação Instituto Brasileiro de Geografia e Estatística. Tabulação avançada do censo demográfico 2000. Resultados preliminares da amostra. Rio de Janeiro: Fundação Instituto Brasileiro de Geografia e Estatística; 2002.
19. Monteiro CA, Conde W. A tendência secular da obesidade segundo estratos sociais: Nordeste e Sudeste do Brasil, 1975-1989-1997. Arq Bras Endocrinol Metab 1999; 43:186-94.

20. Burlandy L, Anjos LA. Acesso a vale-refeição e estado nutricional de adultos beneficiários do Programa de Alimentação do Trabalhador no Nordeste e Sudeste do Brasil, 1997. Cad Saúde Pública 2001; 17:1457-64.

21. Bleil SI. O padrão alimentar ocidental: considerações sobre a mudança de hábitos no Brasil. Cadernos de Debate 1998; VI:1-25.

22. Fischeler C. Gastro-nomia y gastro-anomia: sabiduria del cuerpo y crisis biocultural de la alimentación contemporánea. In: Contreras J, compilador. Alimentación y cultura. Necesidade, gustos y costumbres. Barcelona: Universitat de Barcelona; 1995. p. 357-80.

23. French S, Story M, Jeffery R. Environmental influences on eating and physical activity. Annu Rev Public Health 2001; 22:309-35.

24. Monteiro CA, Mondini L, Costa R. Mudanças na composição e adequação nutricional da dieta familiar nas áreas metropolitanas do Brasil (19881996). Rev Saúde Pública 2000; 34:251-8.

25. Cyrillo DC, Saes MSM, Braga MB. Tendências do consumo de alimentos e o plano Real: uma avaliação para a Grande São Paulo. Planej Polít Públicas 1997; 16:163-95.

26. Castro CM, Peliano AM. Novos alimentos, velhos hábitos e o espaço para ações educativas. In: Castro CM, Coimbra M, organizadores. O problema alimentar no Brasil. São Paulo: Editora da Unicamp/Editora Almed; 1985. p. 195-213.

27. Sichieri R. Dietary patterns and their associations with obesity in the Brazilian City of Rio de Janeiro. Obes Res 2002; 10:42-8.

28. Pereira CVR, Silva CS, Anjos LA. Tendência na disponibilidade de energia e macronutrientes para a população brasileira nos últimos 40 anos. Anais do 12o Seminário de Iniciação Científica. Niterói: Universidade Federal Fluminense; 2002.

29. Wahrlich V, Anjos LA. Aspectos históricos e metodológicos da medição e estimativa da taxa metabólica basal: uma revisão da literatura. Cad Saúde Pública 2001; 18:801-17.

30. Caspersen CJ, Powell KE, Christensen GM. Physical activity, exercise, and physical fitness: definitions and distinctions for health-related reserch. Public Health Rep 1985; 100:126-31.

31. Anjos LA. Obesidade nas sociedades contemporâneas: o papel da dieta e da inatividade física. In: Anais do 3o Congresso Brasileiro de Atividade Física e Saúde. Florianópolis: Universidade Federal de Santa Catarina; 2001. p. 33-4.

32. U.S. Department of Health and Human Services Physical activity and health. A report of the surgeon general. Atlanta: Centers for Disease Control and Prevention, National Center for Chronic Disease Prevention and Health Promotion; 1996.

33. Centers for Disease Control and Prevention. Prevalence of leisure-time and occupational physical activity among employed adults - United States, 1990 MMWR Morb Mortal Wkly Rep 2000; 49:420-4. 
34. Anjos LA. Prevalência da inatividade física no Brasil. In: Anais do 2o Congresso Brasileiro de Atividade Física \& Saúde. Florianópolis: Universidade Federal de Santa Catarina; 1999. p. 58-63.

35. Fundação Instituto Brasileiro de Geografia e Estatística. Censo Demográfico de 1960. VII recenseamento geral do Brasil, série nacional, volume I. Rio de Janeiro: Fundação Instituto Brasileiro de Geografia e Estatística; 1967.

36. Fundação Instituto Brasileiro de Geografia e Estatística. Censo Demográfico de 1980. IX recenseamento geral do Brasil, série nacional, volume I. Rio de Janeiro: Fundação Instituto Brasileiro de Geografia e Estatística; 1983.

37. Fundação Instituto Brasileiro de Geografia e Estatística. Censo Demográfico de 1991. X recenseamento geral do Brasil, série nacional, volume I. Rio de Janeiro: Fundação Instituto Brasileiro de Geografia e Estatística; 1993.

38. Rotenberg S. Práticas alimentares e o cuidado da saúde da criança de baixo-peso [Dissertação de Mestrado]. Rio de Janeiro: Instituto Fernandes Figueira, Fundação Oswaldo Cruz; 1999.

39. Anjos LA. Physical activity estimates from a household survey in Brazil. Med Sci Sports Exerc 2000; 32 (5 Suppl):S188.

40. Crespo CJ, Smit E, Troiano RP, Barlett SJ, Macera CA, Andersen RE. Television watching, energy intake, and obesity in the US children. Arch Pediatr Adolesc Med 2001; 155:360-5.

41. Pickrell D, Schimek P. Growth in motor vehicle ownership and use: Evidence from the Nationwide Personal Transportation Survey. Journal of Transportation and Statistics 1999; 2:1-17.

42. U.S. Census Bureau. Vehicles available and household income in 1999-2000. http:/ / factfinder.cen sus.gov/servlet/QTTable?_ts=50429928110 (acessado em 18/Out/2002).

43. Ministério da Saúde. 10 passos para o peso saudável. http://www.saude.gov.br (acessado em 08/ Mai/2001).

44. Sichieri R, Nascimento S, Moura AS. Algumas reflexões sobre o uso público do conhecimento gerado pela epidemiologia. Physis (Rio J) 2002; 12: 109-20.

45. Phillippi ST, Latterza AR, Cruz ATR, Ribeiro LC. Pirâmide alimentar adaptada: Guia para escolha dos alimentos. Rev Nutr 1999; 12:65-80.

46. Ministério da Saúde/Agência Nacional de Vigilância Sanitária/Universidade de Brasília. Rotulagem nutricional obrigatória: manual de orientação aos consumidores. Brasília: Ministério da Saúde/Agência Nacional de Vigilância Sanitária/ Universidade de Brasília; 2002.
47. $\mathrm{Hu}$ FB, Willett WC. Optimal diets for prevention of coronary heart disease. JAMA 2002; 288:2569-78.

48. Young LR, Nestle M. The contribution of expanding portion sizes to the US obesity epidemic. Am J Public Health 2002; 92:246-9.

49. Rolls BJ, Morris EL, Roe L. Portion size of food affects energy intake in normal-weight and overweight men and women. Am J Clin Nutr 2002; 76:1207-13.

50. Pate RR, Pratt M, Blair SN, Haskell WL, Macera CA, Bouchard C, et al. Physical activity and public health. A recommendation from the Centers for Disease Control and Prevention and the American College of Sports Medicine. JAMA 1995; 273: 402-7.

51. Blair SN, Kohl HW, Paffenbarger RS, Clark DG, Cooper KH, Gibbons LW. Physical fitness and allcause mortality: a prospective study of healthy men and women. JAMA 1989; 273:1093-8.

52. Paffenbarger Jr. RS, Lee IM. Physical activity and fitness for health and longevity. Res Q Exerc Sport 1996; 67 (3 Suppl):11-28.

53. Paffenbarger Jr. RS, Hyde RT, Wing AL, Hsieh C. Physical activity, all-cause mortality, and longevity of college alumni. N Engl J Med 1986; 314:605-13.

54. National Institutes of Health/National American Association for the Study of Obesity. Obesity. The practical guide. Identification, evaluation, and treatment of overweight and obesity in adults. Bethesda: National Institutes of Health; 2000.

55. Williams P. Physical fitness and activity as separate heart disease risk factors: a meta-analysis. Med Sci Sports Exerc 2001; 33:754-61.

56. Food and Nutrition Board/Institute of Medicine of the National Academy. Dietary reference intakes for energy, carbohydrate, fiber, fat, fatty acids, cholesterol, protein, and aminoacids. Washington DC: The National Academy Press; 2002.

57. Vasconcellos MTL, Anjos LA. A simplified method for assessing physical activity level values for a country or study population. Eur J Clin Nutr 2003; 57:1025-33.

58. Erlichman J, Kerbey A, James P. Are current physical activity guidelines adequate to prevent unhealthy weight gain? A scientific appraisal for consideration by an Expert Panel of the International Obesity Task Force. London: International Obesity Task Force; 2001.

59. Food and Agriculture Organization/World Health Organization. Energy requirements. Rome: Food and Agriculture Organization; 2002.

Recebido em 16/Jan/2003

Versão final reapresentada em 08/Ago/2003 Aprovado em 23/Out/2003 